\title{
Ancylostoma caninum
}

National Cancer Institute

\section{Source}

National Cancer Institute. Ancylostoma caninum. NCI Thesaurus. Code C122240.

A species of nematodes in the family Ancylostomatidae. A. caninum infects domestic dogs and wild canids. It occasionally infects cats and humans. It is largely restricted to temperate, tropical, and sub-tropical regions. 